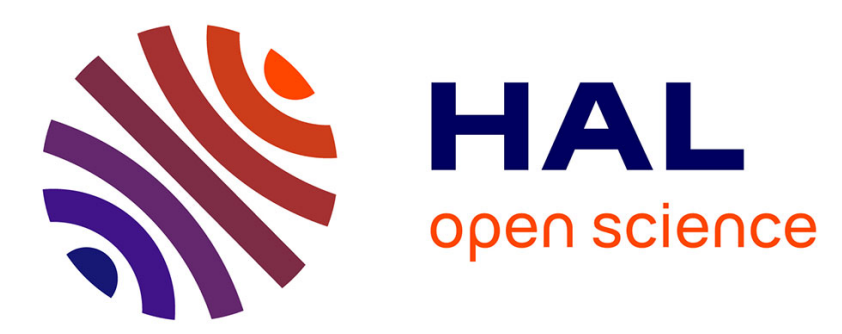

\title{
Opencage radio frequency coil for magnetic resonance imaging
}

\author{
A. Nikulin, J. de Rosny, K. Haliot, B. Larrat, A. Ourir
}

\section{To cite this version:}

A. Nikulin, J. de Rosny, K. Haliot, B. Larrat, A. Ourir. Opencage radio frequency coil for magnetic resonance imaging. Applied Physics Letters, 2019, 114 (5), pp.053503. 10.1063/1.5082245 . hal02393095

\section{HAL Id: hal-02393095 \\ https://hal.science/hal-02393095}

Submitted on 11 Feb 2020

HAL is a multi-disciplinary open access archive for the deposit and dissemination of scientific research documents, whether they are published or not. The documents may come from teaching and research institutions in France or abroad, or from public or private research centers.
L'archive ouverte pluridisciplinaire HAL, est destinée au dépôt et à la diffusion de documents scientifiques de niveau recherche, publiés ou non, émanant des établissements d'enseignement et de recherche français ou étrangers, des laboratoires publics ou privés. 


\section{Opencage radio frequency coil for magnetic resonance imaging}

Cite as: Appl. Phys. Lett. 114, 053503 (2019); https://doi.org/10.1063/1.5082245

Submitted: 20 November 2018 . Accepted: 18 January 2019. Published Online: 05 February 2019

A. Nikulin, J. de Rosny, K. Haliot, B. Larrat, and A. Ourir
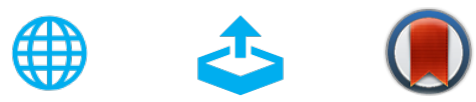

View Online

Export Citation

CrossMark

\section{ARTICLES YOU MAY BE INTERESTED IN}

Enhanced modulation of magnetization in the $\mathrm{Fe}_{3} \mathrm{O}_{4} / \mathrm{MgO} / \mathrm{SrTiO}_{3}$ heterostructure by electric field

Applied Physics Letters 114, 052401 (2019); https://doi.org/10.1063/1.5082256

Multifunctional reflection in acoustic metagratings with simplified design

Applied Physics Letters 114, 053502 (2019); https://doi.org/10.1063/1.5083081

Wideband electromagnetic dynamic acoustic transducers (WEMDATs) for air-coupled ultrasonic applications

Applied Physics Letters 114, 053505 (2019); https://doi.org/10.1063/1.5086383

Lock-in Amplifiers Find out more today

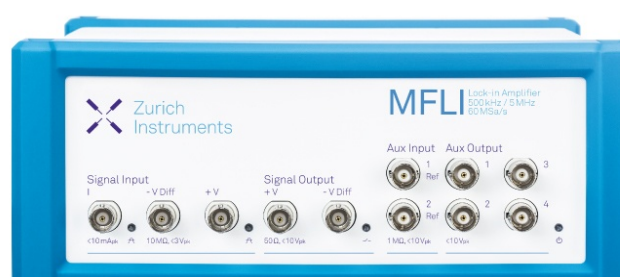

Zurich Instruments 


\title{
Opencage radio frequency coil for magnetic resonance imaging
}

\author{
Cite as: Appl. Phys. Lett. 114, 053503 (2019); doi: 10.1063/1.5082245 \\ Submitted: 20 November 2018 • Accepted: 18 January 2019 . \\ Published Online: 5 February 2019

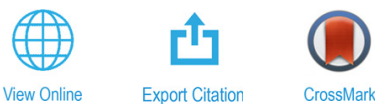

\author{
A. Nikulin,, ${ }^{1, a)}$ J. de Rosny, ${ }^{1}$ K. Haliot$^{2}{ }^{2}$ B. Larrat, ${ }^{2}$ and A. Ourir \\ AFFILIATIONS \\ 1Institut Langevin, ESPCI Paris, CNRS, PSL University, 1 rue Jussieu, 75005 Paris, France \\ ${ }^{2}$ CEA, DRF, JOLIOT, NeuroSpin, UNIRS, Université Paris-Saclay, 91191 Gif-sur-Yvette Cedex, France

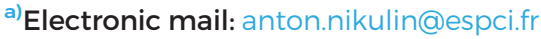

\begin{abstract}
We present a volume radio frequency coil for Magnetic Resonance Imaging that provides access to the region of interest. While the conventional birdcage coil is composed of a periodic array of similar unit cells making a cylindrical structure, the proposed coil, called "opencage," is made of an aperiodic array of metamaterial based unit cells presenting different geometries and characteristics. We develop here a dedicated approach based on Bloch impedance matching and phase balance for the design of the opencage coil. We perform full-wave numerical simulations to validate this concept. An experimental demonstration of the opencage coil for small animal imaging at 7 Tesla is presented. The results of the in-vitro, in-vivo imaging and $B_{1}^{+}$map reconstruction achieved with a preclinical MRI scanner are presented. We show that $\mathrm{B}_{1}^{+}$field homogeneity and amplitude generated by the opencage coil are comparable to those of a conventional birdcage coil of the same size.
\end{abstract}

Published under license by AIP Publishing. https://doi.org/10.1063/1.5082245

MRI is a key apparatus for in-vivo imaging based on the spin precession of some nuclei under a strong static magnetic field at Larmor frequency. Recently, metamaterials have been used in the design of radio frequency (RF) coils for MRI to improve their performances or to provide new interesting features. For instance, metamaterials have been used to control nearfield distribution by exciting the eigenmodes of a wire structure. ${ }^{1}$ They have also been used for dual-nuclei imaging using a single transmit-receive channel of the MRI scanner. The total scan time can be reduced by increasing the sensitivity of various RF coils in the presence of the metasurface. ${ }^{3,4}$

One of the main RF coil geometry in MRI is the so called "birdcage" coil. ${ }^{5,6}$ Introduced in 1985, it is made of interconnected parallel metallic rods (legs) periodically arranged and forming a cylinder. It creates a homogeneous magnetic field and provides a suitable signal-to-noise ratio (SNR) in the region of interest (ROI). However, for some applications, the birdcage cannot be used due to a dense covering of the sample by the legs of this coil. An open access to the ROI can be desirable in many situations. For instance, for brain imaging, it would reduce the discomfort of having the head confined into a narrow cylinder. It could also ease the optical pathway to send visual stimuli to the patients during scans. For multimodal and interventional imaging, it could allow to access the imaged organ with another modality such as a laser, an ultrasound probe, a RF or cryoablation device, a biopsy needle. Being able of opening access to the ROI without significant image degradation compared to the conventional birdcage coil would be really helpful.

This access problem can be resolved by a surface loop coil which is efficient in terms of SNR but in a narrow field of view (FOV) close to the loop. To overcome this limitation, surface coils with complex geometries such as the half of the birdcage coil or the U-shaped birdcage coil ${ }^{8}$ can be used. Although the FOV of these coils are broader, they are still narrower than the FOV of true volume coils like the birdcage coil. Another option would be to decrease the number of legs of a regular birdcage coil to get wider access between legs but unfortunately, this results in a dramatic loss of homogeneity in the ROI.

A birdcage-like coil with a wide aperture for an easy access to ROI necessitates the realization of an aperiodic structure. The design of such a coil requires the adjustment of the current distribution on each leg of the birdcage-like coil. An approach to optimize the current distribution in an elliptical birdcage has been proposed.

In this work, we mix Bloch impedance matching and phase balance of the unit cells to design of a RF coil with a wide 
opening. This so-called "opencage" coil is composed of an array of metamaterial based unit cells. We show numerically and experimentally that the proposed configuration preserves high imaging performance.

Subwavelength periodicity and hybridization of eigenmodes in a birdcage coil allows to consider it as a metamaterial or more exactly as a transmission line (TL) composed of units cells made of inductances and capacitances. Depending on the topology of such a TL, it can be a closed left-handed transmission line (LHTL) or a closed right-handed transmission line (RHTL).

In the present work, we focus on LHTL which is well adapted for an ultra-high field MRI. We propose to design an opencage coil for small animal imaging at 7 Tesla. The operating frequency of the RF coil is the Larmor frequency of ${ }^{1} \mathrm{H}$ at $7 \mathrm{~T}$ $(300.1 \mathrm{MHz})$. This frequency is the highest resonance mode of the close LHTL. The total phase shift of this mode along the LHTL reaches $360^{\circ}$ leading to an ideal cosine current distribution and a homogeneous magnetic field. ${ }^{10}$ For a conventional birdcage with $\mathrm{N}$ unit cells (legs), the phase shift per a unit cell is $2 \pi / N$. In the opencage coil, the cells are not identical. Therefore, the structure should be designed by carefully imposing a total phase shift of all unit cells equal to $2 \pi$ at the desired resonant frequency.

The schematic view of the opencage coil is presented in Fig. 1(a). When considering the fundamental (asymmetric) mode, the electrical models presented in Fig. 1(b) can be used for the unit cell of the two structures (see supplementary material for more details). In the bottom electrical model, $C, L_{r}$, and $L$ are, respectively, the capacitance, the ring effective inductance, and the leg effective inductance. To generate the fundamental mode with a total phase shift of $360^{\circ}$ equivalent to the birdcage one there are 2 conditions. First each of the four (respect. two) cells of the bottom part (respect. top part) should induce a $45^{\circ}$ (respect. $90^{\circ}$ ) phase shift. Second, the Bloch impedance of all the cells should be identical to avoid reflections.

The values of the Bloch impedance $Z_{b}^{i}$ and the phase shift $\Delta \phi_{i}$ of a unit cell (see supplementary material) can be determined by using T-matrix formalism. ${ }^{11}$ It comes

$$
Z_{b}^{i}=\frac{1}{2} \sqrt{Z_{i}^{2}+\frac{2 Z_{i}}{Y_{i}}}, \quad \Delta \phi_{i}=\cos ^{-1}\left(1+Z_{i} Y_{i}\right),
$$

where $Z_{i}$ is the serial impedance and $Y_{i}$ is the parallel admittance. In the case of a left-handed (LH) unit cell these lasts are given by

$$
\mathrm{Z}_{i}=\frac{1}{j \omega \mathrm{C}_{i}}+j \mathrm{~L}_{r, i} \omega, \quad \mathrm{Y}_{i}=\frac{1}{j \omega \mathrm{L}_{i}},
$$

where $C_{i}, L_{i}$, and $L_{i, r}$ are the capacitance, the leg effective inductance, and the ring effective inductance, respectively, of the $i$ th. The effective inductances include the mutual coupling between the legs and the shield, also the mutual coupling between the segments of the rings. ${ }^{12-14}$

According to the dispersion diagram (phase shift) and the Bloch impedance of the LH cell [Figs. 1(c) and 1(d)], the values of the $L_{i}$ and $C_{i}$ has to be changed to achieve the desired phase shift and the constant impedance at $300 \mathrm{MHz}$.

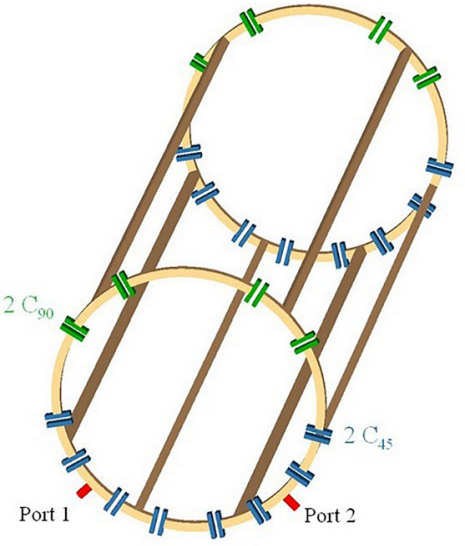

(a)
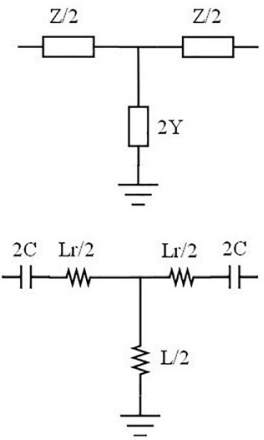

(b)

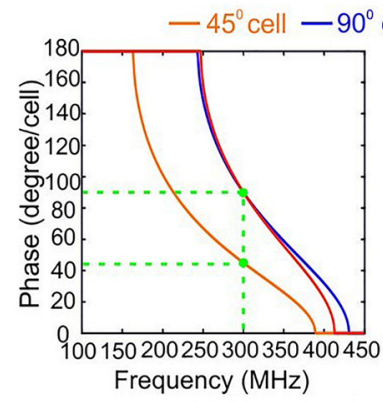

(c)

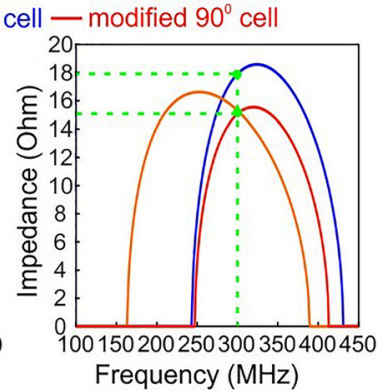

(d)
FIG. 1. Schematic view of the opencage coil (a) and its electrically equivalent unit cell (b). Calculated curves for the $45^{\circ}, 90^{\circ}$, and modified $90^{\circ}$ LHTL cells: (c) the Bloch impedance; and (d) the dispersion diagram.

By using the following values of the capacitance and inductances: $C^{45}=16.94 \mathrm{pF}, \mathrm{L}^{45}=22.96 \mathrm{nH}$, and $\mathrm{L}_{r}^{45}=9.88 \mathrm{nH}$, the phase shift and the Bloch impedance for the $45^{\circ}$ cells are equal to $45.03^{\circ}$ and $15.31 \Omega$, respectively. For the conventional $90^{\circ}$ cells with the same self-inductances (sizes) as $45^{\circ}$ cells, the effective inductances, and the capacitance are the following: $C^{90}=7.63 \mathrm{pF}, L^{90}=19.0 \mathrm{nH}, L_{r}^{90}=17.9 \mathrm{nH}$. That gives a $89.96^{\circ}$ phase shift and a $17.91 \Omega$ Bloch impedance. The size of the metal strips (legs) to obtain these effective inductances is the same for both $45^{\circ}$ and $90^{\circ}$ cells and equal to: $w=w_{1}=2.5 \mathrm{~mm}$ width and $40 \mathrm{~mm}$ length. However, the Bloch impedance of these $90^{\circ}$ cells does not match the $45^{\circ}$ ones.

So the capacitance and inductances values of $90^{\circ}$ cells have been modified to: $\mathrm{C}^{90}=8.3 \mathrm{pF}, \mathrm{L}^{90}=16 \mathrm{nH}$ [the width of the leg $\left(w_{1}\right)$ has to be increased up to $\left.4 \mathrm{~mm}\right], \mathrm{L}_{r}^{90}=17.9 \mathrm{nH}$. With these values, the phase shift is $90.03^{\circ}$ [Fig. 1(c)] and the Bloch impedance is lowered to $15.08 \Omega$ [Fig. 1(d)].

The proposed design [Fig. 2(a)] has been validated by fullwave simulations (CST Microwave studio 2018 Frequency Domain Solver). The geometrical parameters of the opencage coil are presented in the caption of Fig. 2. The opencage coil is placed inside a copper cylinder corresponding to the MRI bore with $90-\mathrm{mm}$ diameter and 1000-mm length. The opencage coil 


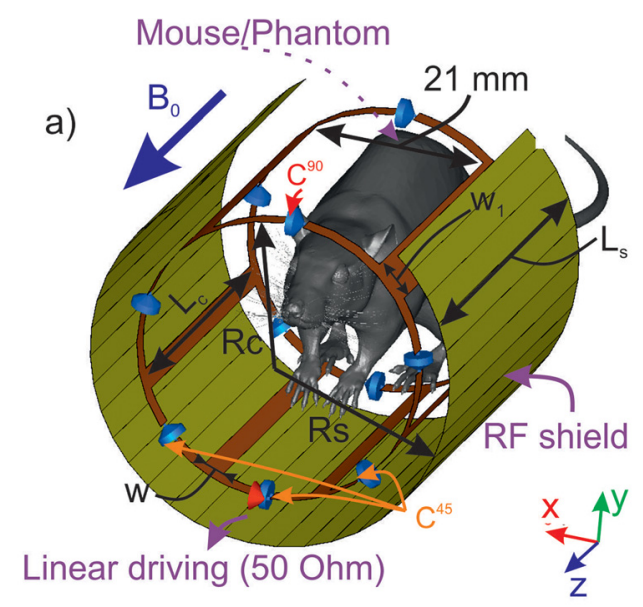

b)

C)

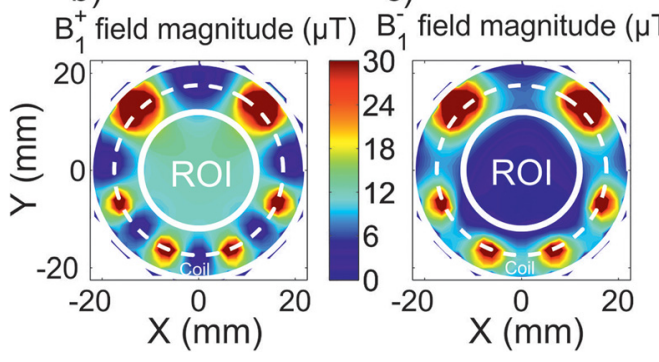

FIG. 2. Design of the opencage coil inside the MRI bore (a). The geometrical parameters of the opencage coil are the following: $R_{s}=22.5 \mathrm{~mm}, R_{c}=17.5 \mathrm{~mm}$, $L_{s}=50 \mathrm{~mm}, L_{c}=40 \mathrm{~mm}, W=2.5 \mathrm{~mm}$, and $w_{1}=5 \mathrm{~mm}$. Here, the width of the modified leg $W_{1}$ is slightly corrected compared to the calculations. Simulated result of the opencage coil driven in a quadrature: (b) $B_{1}^{+}$field in $\mu T$ inside the shield of the opencage coil; and (c) $B_{1}^{-}$field in $\mu T$.

is loaded by a homogeneous cylindrical phantom with relative permittivity and conductivity equal to 45.3 and $0.87 \mathrm{~S} / \mathrm{m}$, respectively. The length of the phantom is $105 \mathrm{~mm}$ and its radius is $13 \mathrm{~mm}$. The values of the capacitors are slightly corrected in comparison with the analytic values in order to obtain the maximum resonance at $300.1 \mathrm{MHz}$. The values are $17.23 \mathrm{pF}$ for the $45^{\circ}$ cells and $8.4 \mathrm{pF}$ for the $90^{\circ}$ cells. Here, the ohmic losses of the capacitors are taken into account with the quality factor $\mathrm{Q}=1000$.

As for birdcage, the structure can generate two counterrotating circularly polarized magnetic modes that are perpendicular to the coil axis. By definition, mode $\mathrm{B}_{1}^{+}$(respect. $\mathrm{B}_{1}^{-}$) is rotating in the same (respect. opposite) direction as the spin precession. ${ }^{15}$ To selectively generate the $\mathrm{B}_{1}^{+}$mode, the opencage coil is driven in quadrature by two $50-\Omega$ ports to excite a circularly polarized mode. The $-20 \mathrm{~dB}$ isolation between the ports due to $90^{\circ}$ phase shift between them confirms that the proper values of the Bloch impedance and phase shift of the unit cells have been reached at the desired frequency of $300 \mathrm{MHz}$ (see supplementary material).

The two circularly polarized magnetic fields $B_{1}$ are observed at the desired frequency in the central $(Z=0)$ transverse plane [Figs. 2(b) and 2(c)]. The field is polarized in this plane. In this case, $B_{1}^{+}$is the maximum, but $B_{1}^{-}$is the minimum which confirming that the opencage behaves as a conventional birdcage coil.

For practical reasons, the opencage coil in the experiment is driven only by a single $50-\Omega$ port. Consequently, an additional full-wave simulation has been performed. Here, the shield of the coil is partially removed to fit the gap between $90^{\circ}$ cells.

For the experimental evaluation, an opencage coil plastic holder has been 3D printed and coppered by tape with $0.035-\mathrm{mm}$ thickness as illustrated in Fig. 3. The opencage coil has been assembled with the following capacitors: $21.3 \mathrm{pF}$ for the $45^{\circ}$ cells and $10.5 \mathrm{pF}$ for the $90^{\circ}$ cells.

The opencage coil is connected through a cable trap to the MRI scanner. A matching circuit with two tunable non-magnetic capacitors is used to achieve the exact desired frequency and the suitable matching level of $\left|\mathrm{S}_{11}\right|<-10 \mathrm{~dB}$ (see supplementary material). The opencage coil has been tuned to $300.1 \mathrm{MHz}$ by adjusting a tunable capacitor $\mathrm{C}_{t}$ mounted instead of one top and front capacitor $\mathrm{C}^{90}$ [Fig. 2(a)]. The tunable nonmagnetic capacitor $C_{m}$ for the matching is connected in series to the feeding coaxial cable (Fig. 3). The opencage coil is first loaded by a cylindrical phantom (13 $\mathrm{mm}$ radius) which is made of a commercial homogeneous liquid (relative permittivity of 45.3 and conductivity of $\sigma=0.87 \mathrm{~S} / \mathrm{m}$ ). Then a living mouse is imaged for in-vivo proof of concept.

The simulated $\mathrm{B}_{1}^{+}$magnetic field at $300.1 \mathrm{MHz}$ inside the phantom is presented in Fig. 4(a). Also, the $B_{1}^{+}$field pattern has been experimentally acquired in the phantom using the Actual Flip Angle (AFI) MRI sequence that was implemented on our Bruker PharmaScan 7T preclinical MRI scanner. ${ }^{16}$ The measured patterns presented on Fig. 4(b) were obtained with the following MRI acquisition parameters: echo time (TE)/repetition time (TR)1/TR2 $=4 / 20 / 100 \mathrm{~ms}$; flip angles $\alpha_{1}=\alpha_{2}=50^{\circ}$; an RF pulse duration of $1 \mathrm{~ms}$; an excitation bandwidth of $14 \mathrm{kHz}$; an FOV of $32 \mathrm{~mm} \times 32 \mathrm{~mm}$; an in plane resolution of $0.5 \mathrm{~mm}$; spoiling gradients with a diffusion coefficient of $2.2 \times 10^{-9} \mathrm{~m}^{2} \mathrm{~s}^{-1}$, and a damping factor of 0.7. Both simulated and experimental patterns are normalized to fit the maximum and minimum of the field in the ROI (phantom). Here, the mismatches between the simulated and measured fields can probably be explained by disorders in the geometry of the opencage coil.
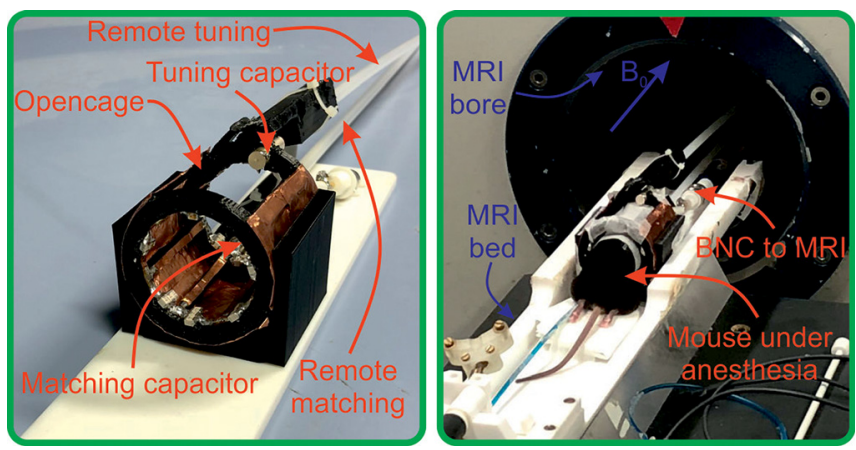

FIG. 3. The assembled prototype of the opencage coil placed in the MRI bed. The setup includes the cable trap and the matching circuit adapted for remote tuning/ matching by rotation of long polyamide rods. 
$\mathrm{B}_{1}^{+}$normalized field to maximum and minimum (inside the ROI) (a.u.) a)

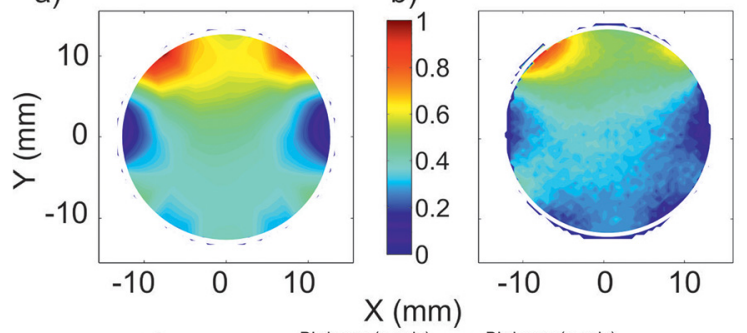

C) $\quad$ Birdcage $(x$-axis) $=-$ Birdcage $(y$-axis $)$

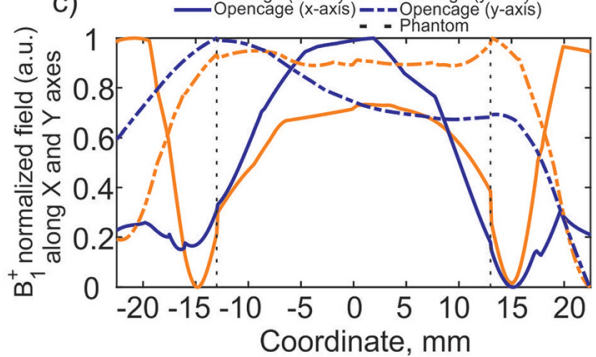

FIG. 4. The simulated and measured opencage coil tuned at $300.1 \mathrm{MHz}$ : (a) normalized $B_{1}^{+}$magnetic field obtained in the simulation; (b) normalized $B_{1}^{+}$magnetic field acquired with MRI; and (c) simulated profiles of the normalized $B_{1}^{+}$magnetic field for the 8-leg birdcage coil and for the opencage coil.

The proposed opencage coil has been compared with the conventional 8-legs birdcage of the same sizes. The profiles of normalized $\mathrm{B}_{1}^{+}$field are presented in Fig. 4(c). Relative standard deviation (SD) of the $\mathrm{B}_{1}^{+}$is estimated in the region where the brain of a mouse is likely to be placed. This region corresponds to the bottom half of the cage in the vicinity of four $45^{\circ}$ cells (legs). The value of relative SD estimated for the opencage coil is equal to $8.8 \%$, while for the birdcage coil this value is $8.3 \%$. These values indicate that the homogeneity of the $\mathrm{B}_{1}^{+}$field for the both coils is comparable in half of the cage volume. When the whole ROI is extended to all the phantom sections, the value of relative SD increases to $20.4 \%$ for the opencage coil while staying almost constant (8.5\%) for the birdcage coil. This increase in SD for the opencage coil is due to the lower density of leg on the top. This is the counterpart of the opening.

The in-vitro images [Figs. 5(a) and 5(b)] have been acquired using the gradient echo sequence (GRE) (TR/TE $=200 / 3 \mathrm{~ms}$, an isotropic voxel of $0.5 \mathrm{~mm}$, an FOV of $32 \mathrm{~mm}$ by $32 \mathrm{~mm}$ by $64 \mathrm{~mm}$, and a flip angle of $30^{\circ}$ ). Moreover, the birdcage coil with 8 legs has been assembled in order to make the comparison with the designed opencage coil. The in-vitro images [Figs. 5(c) and 5(d)] have been also acquired using the same sequence. The injected power has been adjusted in both cases by the Bruker ParaVision software. The SNR of the images has been estimated as a mean signal in the phantom divided by the standard deviation of noise in the ROI which is equal to a matrix of $10 \times 10$ voxels outside the phantom. For the axial image at the center of the coil along the longitudinal axis of the magnet [Fig. 5(a)], the average SNR is equal to 1865, meanwhile for the same plane with the birdcage coil [Fig. 5(c)], the average SNR is equal to 1752. The average SNR

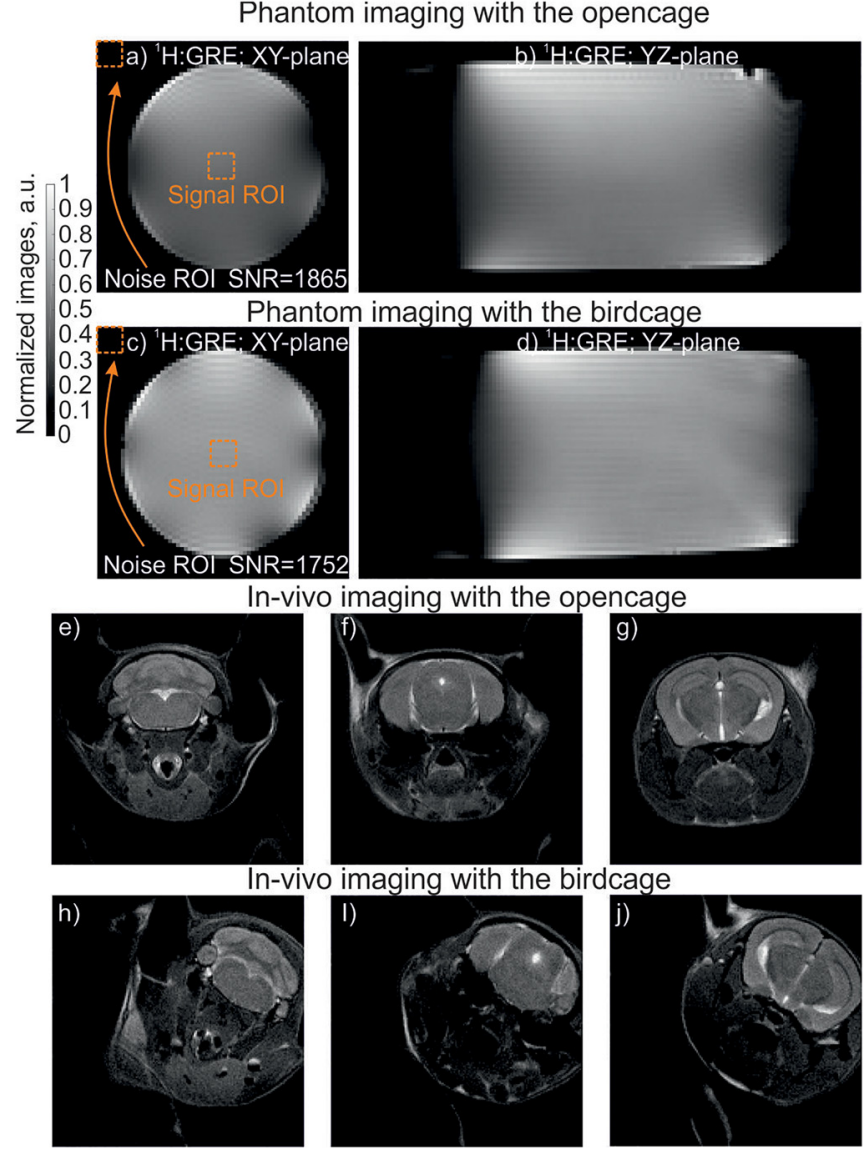

FIG. 5. The normalized images acquired with the opencage coil: (a) the in-vitro image in the central transverse plane; (b) in the central sagittal plane; the normalized images acquired with the birdcage coil: (c) the in-vitro image in the central transverse plane; (d) in the central sagittal plane; (e)-(g) in-vivo images of the mouse brain in several axial slices showing good SNR and good spatial homogeneity. The in-vivo images have been acquired with the opencage coil; and (h) and (i) in-vivo images acquired with the 8-leg birdcage coil.

of both images are comparable, moreover both coils are demonstrating sufficient homogeneity of the signal in the broad FOV.

The proposed opencage coil has then been tested in-vivo on an anesthetized mouse. The acquired images at several transverse slices are presented in Figs. 5(e)-5(g) for the opencage coil and Figs. 5(h)-5(i) for the conventional 8-legs birdcage coil. For the in-vivo imaging, the spin echo sequence is used (TR/TE $=6200 / 30 \mathrm{~ms}$, an anisotropic voxel of $0.125 \mathrm{~mm}^{2} \times 0.5 \mathrm{~mm}$, an FOV of $20 \mathrm{~mm}$ by $20 \mathrm{~mm}$ by $32 \mathrm{~mm}$, the number of averages of 14 , and the flip angle of $90^{\circ}$ ). Eventually, the signal level in the ROI is suitable, meantime the opencage coil provides sufficient FOV to scan a brain or a half body of small animals.

We have presented a RF coil for MRI, called the opencage coil-that provides easy access to the imaged sample. We have developed an approach based on the Bloch impedance matching and the phase adjusting to design the proposed RF coil. The parameters of the opencage coil have been determined 
analytically, tested numerically, and confirmed experimentally on the bench and in the MRI. In-vitro and in-vivo images have been acquired. The opencage coil demonstrates sufficient signal homogeneity and suitable FOV for MR imaging of small animals at 7T. Compared to an 8-leg birdcage coil, the homogeneity of signal is sufficient. At the same time, the SNR of images acquired by both coils are comparable, while the opencage coil provides a wide access that can be useful in an interventional MRI studies in small animals.

See supplementary material for a detailed description of the lumped element network model and for the simulated and measured S-parameters spectra of the opencage coil.

This project has received funding from the European Union's Horizon 2020 research and innovation program under Grant Agreement No. 736937. This work is supported by LABEX WIFI (Laboratory of Excellence within the French Program "Investments for the Future") under references ANR10-LABX-24 and ANR-10-IDEX-0001-02 PSL.

\section{REFERENCES}

${ }^{1}$ C. Jouvaud, R. Abdeddaim, B. Larrat, and J. de Rosny, "Volume coil based on hybridized resonators for magnetic resonance imaging," Appl. Phys. Lett. 108, 023503 (2016).

${ }^{2}$ A. Hurshkainen, A. Nikulin, E. Georget, B. Larrat, D. Berrahou, L. Neves, P. Sabouroux, S. Enoch, I. Melchakova, P. Belov, S. Glybovski, and R. Abdeddaim, "A novel metamaterial-inspired RF-coil for preclinical dualnuclei MRI," Sci. Rep. 8, 9190 (2018).

${ }^{3}$ A. P. Slobozhanyuk, A. N. Poddubny, A. J. E. Raaijmakers, C. A. T. van den Berg, A. V. Kozachenko, I. A. Dubrovina, I. V. Melchakova, Y. S. Kivshar, and P. A. Belov, "Enhancement of magnetic resonance imaging with metasurfaces," Adv. Mater. 28, 1832-1838 (2016).
${ }^{4}$ E. I. Kretov, A. V. Shchelokova, and A. P. Slobozhanyuk, "Impact of wire metasurface eigenmode on the sensitivity enhancement of MRI system," Appl. Phys. Lett. 112, 033501 (2018).

${ }^{5}$ C. E. Hayes, W. A. Edelstein, J. F. Schenck, O. M. Mueller, and M. Eash, "An efficient, highly homogeneous radiofrequency coil for whole-body NMR imaging at 1.5 T," J. Magn. Reson. 63, 622-628 (1985).

${ }^{6} \mathrm{C}$. E. Hayes, "The development of the birdcage resonator: A historical perspective," NMR Biomed. 22, 908-918 (2009).

${ }^{7}$ D. Ballon, M. C. Graham, S. Miodownik, and J. A.Koutcher, "A 64 MHz halfbirdcage resonator for clinical imaging," J. Magn. Reson. 90, 131-140 (1990).

${ }^{8}$ A. M. J. Hudson, W. Kockenberger, and R. W. Bowtell, "Open access birdcage coils for microscopic imaging of plants at 11.7 T," Magn. Reson. Mater. Phys., Biol. Med. 10, 69-74 (2000).

${ }^{9} \mathrm{~S}$. Li, C. M. Collins, B. J. Dardzinski, C. L. Chin, and M. B. Smith, "A method to create an optimum current distribution and homogeneous B1 field for elliptical birdcage coils," Magn. Reson. Med. 37, 600-608 (1997).

${ }^{10} \mathrm{~J}$. Mispelter, M. Lupu, and A. Briguet, NMR Probeheads for Biophysical and Biomedical Experiments: Theoretical Principles and Practical Guidelines, 2nd ed. (Imperial College Press, 2015).

${ }^{11} \mathrm{C}$. Caloz and T. Itoh, Electromagnetic Metamaterials: Transmission Line Theory and Microwave Applications (John Wiley and Sons Ltd, 2006).

${ }^{12}$ F. W. Grover, Inductance Calculations, Working Formulas and Tables (Dover publications, 1973).

${ }^{13}$ C.-L. Chin, C. M. Collins, S. Li, B. J. Dardzinski, and M. B. Smith, "BirdcageBuilder: Design of specified-geometry birdcage coils with desired current pattern and resonant frequency," Concepts Magn. Reson. 15(2), 156-163 (2002).

${ }^{14}$ R. Pascone, T. Vullo, J. Farrelly, and P. T. Cahill, "Explicit treatment of mutual inductance in eight-column birdcage resonators," Magn. Reson. Imaging 10, 401-410 (1992).

${ }^{15}$ M. V. Vaidya, C. M. Collins, D. K. Sodickson, R. Brown, G. C. Wiggins, and R. Lattanzi, "Dependence of $B_{1}^{+}$and $B_{1}^{-}$field patterns of surface coils on the electrical properties of the sample and the MR operating frequency," Concepts Magn. Reson., Part B 46(1), 25-40 (2016).

${ }^{16} \mathrm{~K}$. Nehrke, "On the steady-state properties of actual flip angle imaging (AFI)," Magn. Reson. Med. 61, 84-92 (2009). 